\title{
An Explicit Marching-on-in-time Scheme for Solving the Kirchhoff Integral Equation
}

\author{
Rui Chen, Sadeed Bin Sayed, and Hakan Bagci \\ Computer, Electrical and Mathematical Science and Engineering Division \\ King Abdullah University of Science and Technology (KAUST), Thuwal, 23955-6900, Saudi Arabia \\ e-mails: \{rui.chen, sadeed.sayed, hakan.bagci\}@kaust.edu.sa
}

\begin{abstract}
An explicit marching-on-in-time scheme for solving the Kirchhoff integral equation enforced on an acoustically rigid scatterer is proposed. The unknown velocity potential introduced on the surface of scatterer is expanded using unit pulse functions in space and Lagrange polynomial interpolation functions in time. The resulting system is cast in the form of an ordinary differential equation and then integrated numerically in time using a predictor-corrector scheme to obtain the unknown expansion coefficients. Numerical results demonstrate that the time step size required by the proposed explicit scheme to ensure an accurate and stable solution is as large as that used by its implicit counterpart.
\end{abstract}

\section{INTRODUCTION}

Transient analysis of acoustic scattering from rigid objects is oftentimes analyzed by solving the time dependent Kirchhoff (surface) integral equation (TDKIE) [1]. This is due to fact that integral equation solvers use a Green function based formulation, which makes them immune to numerical phase dispersion and permits them to enforce the radiation condition without the need to truncate the computation domain by an (approximate) absorbing boundary condition [1]. The most well-known method for solving the TDKIE of acoustics is the marching-on-in-time (MOT) scheme [1]. Depending on the type of spatial and temporal basis functions used for expanding the unknown velocity potential introduced on the scatterer surface and the time step size, the MOT scheme can be either explicit or implicit [2], [3], [4]. Although traditional explicit MOT schemes do not require matrix inversion during time marching, they use smaller time steps for a stable solution in comparison to their implicit counterparts. Recently, a novel quasi-explicit MOT scheme has been developed to overcome this bottleneck [2]. This scheme uses Rao-Wilton-Glisson (RWG) functions [5] as the spatial basis and testing functions to discretize the time domain magnetic field integral equation (TD-MFIE) in unknown current density. The resulting semidiscrete system is cast in the form of an ordinary differential equation (ODE), which is then integrated in time using a $\mathrm{PE}(\mathrm{CE})^{\mathrm{m}}$-type linear multistep scheme to obtain the unknown coefficients of the RWG basis functions. This quasi-explicit approach requires the solution of a system with a sparse and well-conditioned Gram matrix at each time step, which is carried out using an iterative solver.

In this work, a fully explicit MOT scheme is developed for solving the TDKIE for analyzing transient acoustic scattering from rigid objects. Unlike the above quasi-explicit solver, pulse functions are used in space to discretize the TDKIE (instead of RWG basis functions). Since each pulse function resides on only one triangle, the resulting Gram matrix is diagonal. This eliminates the need for iterative solution at every time step and significantly increases the efficiency of time marching. Numerical results demonstrate that the proposed solver can use the same time step size as its implicit counterpart without sacrificing from accuracy or stability.

\section{FORMULATION}

Let $S$ denote the surface of an acoustically rigid object residing in an unbounded homogeneous medium. The scatterer is excited by a band-limited incident field with velocity potential $\varphi^{\text {inc }}(\mathbf{r}, t)$ that is vanishingly small for $t \leq 0$ and $\forall \mathbf{r} \in S$. The total velocity potential $\varphi(\mathbf{r}, t)$ introduced on $S$ is related to $\varphi^{\text {inc }}(\mathbf{r}, t)$ through the TDKIE [1]:

$$
\begin{aligned}
\frac{1}{2} \partial_{t} \varphi(\mathbf{r}, t) & =\partial_{t} \varphi^{\text {inc }}(\mathbf{r}, t) \\
& +\partial_{t} \int_{S} \varphi\left(\mathbf{r}^{\prime}, t\right) \hat{\mathbf{n}}\left(\mathbf{r}^{\prime}\right) \cdot \nabla^{\prime} \frac{\delta(t-R / c)}{4 \pi R} d \mathbf{r}^{\prime} .
\end{aligned}
$$

Here, $R=\left|\mathbf{r}-\mathbf{r}^{\prime}\right|$ is the distance between observation point $\mathbf{r}$ and source point $\mathbf{r}^{\prime}, \hat{\mathbf{n}}\left(\mathbf{r}^{\prime}\right)$ is the unit outward normal vector at $\mathbf{r}^{\prime}$, and $c$ is the speed of sound in the background medium.

To numerically solve (1), first $S$ is discretized into $N_{\mathrm{s}}$ flat triangular patches and $\varphi(\mathbf{r}, t)$ is expanded using pulse functions $f_{n}(\mathbf{r})$ as

$$
\varphi(\mathbf{r}, t)=\sum_{n=1}^{N_{\mathrm{s}}}\{\mathbf{I}(t)\}_{n} f_{n}(\mathbf{r}) .
$$

Here, $\{\mathbf{I}(t)\}_{n}$ is time-dependent unknown coefficient of $f_{n}(\mathbf{r})$, and $f_{n}(\mathbf{r})=1$ for $\mathbf{r} \in S_{n}, f_{n}(\mathbf{r})=0$ for $\mathbf{r} \notin S_{n}$, where $S_{n}$ is the surface of $n^{\text {th }}$ triangular patch. Inserting (2) into 1 and point testing the resulting equation at the centers of the patches $\mathbf{r}_{m}, m=1, \ldots, N_{\mathrm{s}}$, yield a time-dependent linear matrix system. Sampling this system at times $t=k \Delta t, k=$ $1, \ldots, N_{\mathrm{t}}$, and using Lagrange polynomial functions $T(t)$ [6] to facilitate the computation of the retarded time integrals yield

$$
\dot{\mathbf{I}}_{k}=\mathbf{V}_{k}^{\mathrm{inc}}+\sum_{l=1}^{k} \mathbf{Z}_{k-l} \mathbf{I}_{l}, k=1, \ldots, N_{\mathrm{t}}
$$


where $\left\{\mathbf{I}_{k}\right\}_{n}=\{\mathbf{I}(k \Delta t)\}_{n},\left\{\dot{\mathbf{I}}_{k}\right\}_{n}=\left\{\left.\partial_{t} \mathbf{I}(t)\right|_{t=k \Delta t}\right\}_{n}$, and the entries of $\mathbf{G}, \mathbf{V}_{k}^{\text {inc }}$, and $\mathbf{Z}_{k-l}$ are given by

$$
\begin{gathered}
\{\mathbf{G}\}_{m n}=\frac{1}{2} \delta_{m n} \\
\left\{\mathbf{V}_{k}^{\mathrm{inc}}\right\}_{m}=\left.\partial_{t} \varphi^{\mathrm{inc}}\left(\mathbf{r}_{m}, t\right)\right|_{t=k \Delta t} \\
\left\{\mathbf{Z}_{k-l}\right\}_{m n}=\frac{1}{4 \pi} \int_{S_{n}} \hat{\mathbf{n}}\left(\mathbf{r}^{\prime}\right) \\
\cdot \frac{\mathbf{R}}{R^{3}}\left[\left.\partial_{t} T_{l}(t)\right|_{t=k \Delta t-R / c}+\left.\frac{R}{c} \partial_{t}^{2} T_{l}(t)\right|_{t=k \Delta t-R / c}\right] d \mathbf{r}^{\prime} .
\end{gathered}
$$

Here, $\mathbf{R}=\mathbf{r}_{m}-\mathbf{r}^{\prime}, R=|\mathbf{R}|$, and $T_{l}(t)=T(t-l \Delta t)$. The ODE system (3) is integrated in time using a $\mathrm{PE}(\mathrm{CE})^{\mathrm{m}}$ method enhanced with a successive-over-relaxation (SOR) scheme [7] to obtain the unknown expansion coefficients. The resulting time marching scheme requires the solution of the matrix system (3) at every time step. This is a trivial task since the $\mathbf{G}^{-1}$ is a diagonal matrix with all entries equal to 2 .

\section{NumericAl RESUlts}

In this section, the proposed explicit scheme and its implicit counterpart are used for analyzing transient acoustic scattering from a rigid unit sphere centered at the origin in a background medium with $c=343 \mathrm{~m} / \mathrm{s}$. The sphere is excited by a plane wave with velocity potential $\varphi^{\text {inc }}(\mathbf{r}, t)=G\left(t-z / c-t_{\mathrm{p}}\right)$, where $G(t)=\cos \left(2 \pi f_{0} t\right) e^{-t^{2} / 2 \sigma^{2}}, t_{\mathrm{p}}$ is the time delay, $\sigma$ is a measure of temporal width of the Gaussian pulse, and $f_{0}$ is its center frequency. In the simulations carried out here, $T(t)$ is a third-order Lagrange polynomial, the $\mathrm{PE}(\mathrm{CE})^{\mathrm{m}}$ scheme uses the predictor and corrector coefficients derived in [8], the SOR factor is set to 0.6 , the implicit scheme uses the transpose-free quasi-minimal residual (TFQMR) method [9] to iteratively solve the MOT system at every time step, and the convergence thresholds for the implicit scheme (iterative solution) and explicit scheme (corrector updates) are both set to $10^{-13}$.

The sphere is discretized into 10616 flat triangle patches, and $f_{0}=200 \mathrm{~Hz}, \sigma=7.639 \mathrm{~ms}, t_{\mathrm{p}}=45.834 \mathrm{~ms}, \Delta t=$ $0.1538 \mathrm{~ms}$, and $N_{\mathrm{t}}=975$. Fig. 1 compares the magnitude of the expansion coefficient computed by the explicit and implicit schemes at the point $(0.060,0.978,0.169 \mathrm{~m})$. The results agree very well. Fig. 2 compares the scattering velocity potential in the farfield region obtained from the Mie series and the solution computed by the explicit scheme at $f=200 \mathrm{~Hz}$ for $\theta=\left[0^{\circ}, 180^{\circ}\right]$. The figure clearly demonstrates the accuracy of the proposed explicit scheme.

\section{REFERENCES}

[1] A. A. Ergin, B. Shanker, and E. Michielssen, "Analysis of transient wave scattering from rigid bodies using a Burton-Miller approach", J. Acoust. Soc. Am., vol. 106, no. 5, pp. 2396-2404, Nov. 1999.

[2] H. A. Ulku, H. Bagci, and E. Michielssen, "Marching on-in-time solution of the time domain magnetic field integral equation using a predictorcorrector scheme", IEEE Trans. Antennas Propag., vol. 61, no. 8, pp. 4120-4131, Aug. 2013.

[3] A. Al-Jarro, M. A. Salem, H. Bagci, T. M. Benson, P. Sewell, and A. Vukovic, "Explicit solution of the time domain volume integral equation using a stable predictor-corrector scheme", IEEE Trans. Antennas Propag., vol. 60, no. 11, pp. 5203-5214, Nov. 2012.

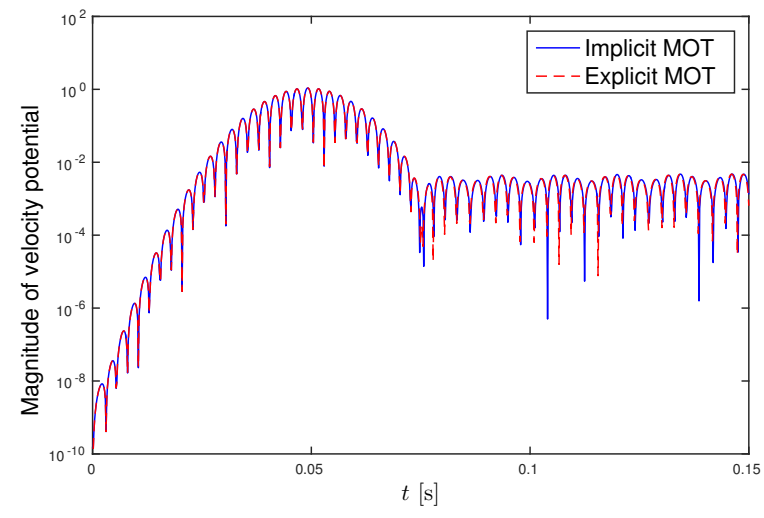

Fig. 1. Potential expansion coefficient computed by the implicit and explicit MOT schemes at the point $(0.060,0.978,0.169 \mathrm{~m})$.

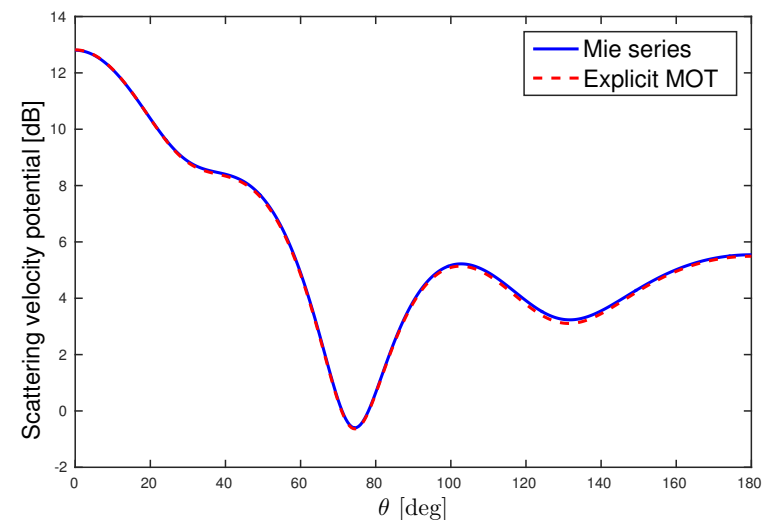

Fig. 2. Scattering velocity potential in the farfield region obtained from the Mie series and the MOT solution at $f=200 \mathrm{~Hz}$ for $\theta=\left[0^{\circ}, 180^{\circ}\right]$.

[4] S. B. Sayed, H. A. Ulku, and H. Bagci, "A stable marching on-in-time scheme for solving the time-domain electric field volume integral equation on high-contrast scatterers", IEEE Trans. Antennas Propag., vol. 63, no. 7, pp. 3098-3110, July 2015.

[5] S. M. Rao, D. R. Wilton, and A. W. Glisson, "Electromagnetic scattering by surfaces of arbitrary shape", IEEE Trans. Antennas Propag., vol. 30, no. 3, pp. 408-418, May. 1982.

[6] H. Bagci, A. E. Yilmaz, V. Lomakin, and E. Michielssen, "Fast solution of mixed-potential time-domain integral equations for half-space environments", IEEE Trans. Geosci. Remote Sens., vol. 43, no. 2, pp. 269-279, Feb. 2005.

[7] S. B. Sayed, H. A. Ulku, and H. Bagci, "Stable quasi-explicit MOT solver for the time domain volume electric field integral equation", in Proc. Appl. Comput. Electromagn. Symp., pp. 416-420, Mar. 2014.

[8] A. Glaser and V. Rokhlin, "A new class of highly accurate solvers for ordinary differential equations", J. Sci. Comput., vol. 38, no. 3, pp. 368399, Mar. 2009.

[9] R. W. Freund, "A transpose-free quasi-minimal residual algorithm for non-Hermitian linear systems", SIAM J. Sci. Comput., vol. 14, no. 2, pp. 470-482, Mar. 1993. 